\title{
IPT in Postpartum Depression
}

Goksen Yuksel ${ }^{1^{*}}$, Nazan Aydın ${ }^{1}$ and Oguz Omay ${ }^{2}$

${ }^{1}$ Psychiatrist, Professor of Psychiatry, 15th Women's Psychiatry Ward, Prof. Dr. Mazhar Osman Bakirkoy Research and Training Hospital for Psychiatry, Neurology and Neurosurgery, Istanbul, Turkey

${ }^{2}$ Psychiatrist, La Teppe Hospital, Tain I'hermitage, France

*Corresponding author: Goksen Yuksel, Psychiatrist, Professor of Psychiatry, 15th Women's Psychiatry Ward, Prof. Dr. Mazhar Osman Bakirkoy Research and Training Hospital for Psychiatry, Neurology and Neurosurgery, Istanbul, Turkey, E-mail: drgoksenyuksel@gmail.com

Received date: October 8, 2015; Accepted date: November 12, 2015; Published date: November 20, 2015

Copyright: (c) 2015 Yuksel G, et al. This is an open-access article distributed under the terms of the Creative Commons Attribution License, which permits unrestricted use, distribution, and reproduction in any medium, provided the original author and source are credited.

\begin{abstract}
Postpartum depression, causing women great suffering, has negative consequences for their social relationships as well as for the development of their infants. Treatment of perinatal depression is vital, otherwise, it may have negative consequences such as chronicity or the psychosocial growth retardation of the infant. Especially depressed mothers face the dilemma of medical treatment versus psychotherapy, although they have better longterm outcomes when used together in clinical practice. Interpersonal Therapy (IPT), a time-limited, dynamically informed and present-focused psychotherapy which targets to improve the interpersonal functioning of patients is a convenient option for the patients mostly face the role transitions and need interpersonal support related to motherhood.
\end{abstract}

Keywords: Postpartum; Depression; Interpersonal psychotherapy

\section{Introduction}

As shown in the literature before, perinatal mental disorders bear potential risks for the (expectant) mother and the offspring [1]. Mostly depression as well as the rest of the psychiatric diseases can take place during this special time. Approximately $13 \%$ of women fulfill the diagnostic criteria for a major depressive episode with postpartum onset [2]. It is known that untreated depression during pregnancy is an important predictor of postpartum depression and treating depression during pregnancy plays a preventative role in postpartum depression [3].

When it comes to psychiatric treatment with psychotrophics, it needs to be evaluated in terms of risk factors and treatment protocols. As the necessity to use medicine during pregnancy or breastfeeding period, when both the mothers and the psychiatrists have concerns, mothers are targeted to be treated on one hand while concerning to minimize the possibility of the unborn or infant damage on the other. Even if not breastfeeding, many women do think prescriptions as second line choise, mostly because of the the fear of "potential longterm side effects".

During perinatal period, not only the multiple biological changes but also role transitions related to motherhood do take place [4]. As many biopsychosocial varients make it so individual for the new mother [5], the most important one is the interpersonal context. Inadequate or lack of perceived interpersonal support and the consequences of it makes it hard to cope with.

Interpersonal therapy (IPT), developed in the 1970s, is a timelimited, dynamically informed and present-focused psychotherapy which targets to improve the interpersonal functioning of patients [6]. Surprisingly, originally developed to be a placebo psychotherapy, soon found to have therapeutic effect [7]. It took a while for it to be used worldwide and admitted as an efficient, empirically tested psychotherapy. IPT, mainly underlined its approach to mood disorders that enhances the importance of psychosocial factors of postpartum depression [8].

Fifty-three non-treatment seeking, pregnant patients underwent "Enhanced IPT-B" is a multicomponent model of care designed to treat antenatal depression and consists of an engagement session, followed by eight acute IPT-B sessions before the birth and maintenance IPT up to six months postpartum. They were assessed before and after treatment on depression diagnoses, depressive symptoms, and social functioning showing that ameliorates depression during pregnancy and prevents depressive relapse and improves social functioning up to six months postpartum [9].

In a study run by Pearlstein et al. in 2006; 12 weeks of outcomes between sertraline alone, IPT alone, or combined (sertraline+IPT) were compared whereas no statistical differences were found between the three groups. The most relevant finding was that women in the study at first depressive episode, when informed of the advantages and concerns of both IPT and sertraline, selected IPT with or without the addition of sertraline.

IPT can also be conceptualized in group settings. In a randomized controlled trial, 50 postpartum depression patients' outcomes who were counted in the 8-week IPT-G group and "treatment as usual" (TAU) group like antidepressants, natural remedies, non-directive counseling, and other interventions were compared [10]. Patients treated with IPT-G (consisted of two individual sessions, eight group therapy sessions ( $2 \mathrm{~h}$ duration) and an additional $2 \mathrm{~h}$ partner's evening) improved significantly more than those treated with TAU and showed a sustained improvement after 3 months. As the patients were also assessed in terms of marital functioning and perceptions of the mother-newborn relationship, women who received IPT-G displayed significant improvement.

Another example of interpersonal-psychotherapy-oriented contex, a postnatal psychoeducation programme were run by the researchers showing to be effective in the Chinese first-time mothers who had significantly fewer depressive symptoms in the 6 week follow up [11]. 
Page 2 of 2

Like the other "special groups" of psychiatry, perinatal patients do need extra care and treatment tools because of the precision of the period also including the infant. IPT, which were proposed to be one of the first-line treatments for PPD (at least for mild or moderate forms of depression) will be providing improvements in interpersonal context [12]. Especially mothers who regret to take medication concerning drug exposure during breastfeeding or mothers who did not get proper improvement with drug therapy during this period will be good candidates for IPT.

\section{References}

1. Brockington I, Chandra P, Dubowitz H, Jones D, Moussa S, et al. (2011) WPA guidance on the protection and promotion of mental health in children of persons with severe mental disorders. World Psychiatry 10: 93-102.

2. Dietz PM, Williams SB, Callaghan WM, Bachman DJ, Whitlock EP, et al. (2007) Clinically identified maternal depression before, during, and after pregnancies ending in live births. Am J Psychiatry 164: 1515-1520.

3. Yazici E, Kirkan TS, Aslan PA, Aydin N, Yazici AB (2015) Untreated depression in the first trimester of pregnancy leads to postpartum depression: high rates from a natural follow-up study. Neuropsychiatr Dis Treat 11: 405-411.

4. Miniati M, Callari A, Calugi S, Rucci P, Savino M, et al. (2014) Interpersonal psychotherapy for postpartum depression: a systematic review. Arch Womens Ment Health 17: 257-268.
5. Martini J, Petzoldt J, Einsle F, Beesdo-Baum K, Höfler M, et al. (2015) Risk factors and course patterns of anxiety and depressive disorders during pregnancy and after delivery: a prospective-longitudinal study. J Affect Disord 175: 385-395.

6. Klerman GL, Dimascio A, Weissman M, Prusoff B, Paykel ES (1974) Treatment of depression by drugs and psychotherapy. Am J Psychiatry 131: 186-191.

7. O'Hara MW, Stuart S, Gorman LL, Wenzel A (2000) Efficacy of interpersonal psychotherapy for postpartum depression. Arch Gen Psychiatry 57: 1039-1045.

8. Meltzer-Brody S (2011) New insights into perinatal depression: pathogenesis and treatment during pregnancy and postpartum. Dialogues Clin Neurosci 13: 89-100.

9. Grote NK, Swartz HA, Geibel SL, Zuckoff A, Houck PR, et al. (2009) A randomized controlled trial of culturally relevant, brief interpersonal psychotherapy for perinatal depression. Psychiatr Serv 60: 313-321.

10. Mulcahy R, Reay RE, Wilkinson RB, Owen C (2010) A randomised control trial for the effectiveness of group Interpersonal Psychotherapy for postnatal depression. Arch Womens Ment Health 13: 125-139.

11. Gao LL, Xie W, Yang X, Chan SW (2015) Effects of an interpersonalpsychotherapy-oriented postnatal programme for Chinese first-time mothers: a randomized controlled trial. Int J Nurs Stud 52: 22-29.

12. Pearlstein TB, Zlotnick C, Battle CL, Stuart S, O'Hara MW, et al. (2006) Patient choice of treatment for postpartum depression: a pilot study. Arch Womens Ment Health 9: 303-308. 